\title{
BMJ Open The relationship between suicidal ideation and symptoms of depression in Japanese workers: a cross-sectional study
}

\author{
Takeaki Takeuchi, Mutsuhiro Nakao
}

To cite: Takeuchi T, Nakao M. The relationship between suicidal ideation and symptoms of depression in Japanese workers: a crosssectional study. BMJ Open 2013;3:e003643

doi:10.1136/bmjopen-2013003643

- Prepublication history for this paper is available online. To view these files please visit the journal online (http://dx.doi.org/10.1136/ bmjopen-2013-003643).

Received 24 July 2013 Revised 27 October 2013 Accepted 1 November 2013

CrossMark

Department of Hygiene and Public Health and Division of Psychosomatic Medicine, Teikyo University School of Medicine, Tokyo, Japan

Correspondence to Dr Takeaki Takeuchi; takeakij@post.harvard.edu

\section{ABSTRACT}

Objectives: The prevalence of suicidal ideation and predictors for suicidal ideation among Japanese workers is unknown, although a previous study reported a $30 \%$ prevalence rate of suicidal ideation in a psychosomatic clinical setting. Hence, we evaluated the prevalence of suicidal ideation and its relationship with depressive symptoms among Japanese workers. Methods: For this purpose, a cross-sectional design was used. Major depressive disorder (MDD) and suicidal ideation in 1266 workers (1100 men and 166 women, aged 20-69 years) were assessed through clinical interviews conducted in accordance with the fourth edition of the Diagnostic and Statistical Manual of Mental Disorders.

Results: A total of 34 and 70 participants were diagnosed with suicidal ideation and MDD, respectively. Suicidal ideation was especially prevalent in 40-year-olds to 49-year-olds. Six of the eight symptoms of MDD (depressive mood, loss of interest, weight loss, psychomotor agitation, worthlessness and concentration loss) were related to suicidal ideation. Depressive mood had the strongest relationship with suicidal ideation, followed by worthlessness and concentration loss. Worthlessness had the highest area under the curve in predicting suicidal ideation, followed by concentration loss and depressive mood.

Conclusions: We conclude that MDD symptomsparticularly depressive mood, worthlessness and concentration loss-are potential predictors of suicidal ideation in Japanese workers.

Although Japan has the highest global ranking for longevity, it also has one of the highest suicide rates, being ranked ninth for men and third for women in the world. ${ }^{1}$ From 1995 to 1998, the incidence of reported suicides rose dramatically from 17 to $25 / 100000$ people, and that high rate continued even in 2010. ${ }^{2}{ }^{3}$ Since 2005, the Japanese Cabinet Office and the Ministry of Health, Labour and Welfare have been attempting to reduce the suicide rate;

\section{Strengths and limitations of this study}

- This is the first evaluation of suicidal ideation among workers in Japan, which has one of the highest rates of suicide worldwide.

- Assessment of major depressive disorder (MDD) including suicidal ideation were made by clinical interviews based on the Diagnostic Statistical Manual of Mental Disorders Fourth Edition, Text Revision.

- Results do not directly imply that symptoms of MDD and suicidal ideation are part of a clinical continuum because of its cross-sectional nature.

- Results do not directly represent the general population of Japanese workers because of the small sample size.

however, the suicide rate in Japan has not decreased.

To reduce the number of suicides, collaborative care, that is, structured care involving a greater role of non-medical specialists for treating depression has shown a potential in both improving depression outcomes and decreasing suicide risk. ${ }^{4}$ However, with the exception of psychiatrists or psychological specialists, most doctors find it difficult to diagnose depression because of the complexities involved in administering clinical interviews. In addition, doctors must pay attention to those who do not perfectly match the diagnostic criteria. ${ }^{5}$ If depression is not diagnosed appropriately, it might be difficult to find the pathway that leads to suicide.

In addition to investigate the potential for depression, it would be much simpler and more useful to determine the presence of suicidal ideation in individuals to assess their suicide risk. Suicidal ideation, which refers to wishing that one's life would end or thoughts of harming or killing oneself, represents an important phase in the suicidal process and often precedes suicidal attempts or death by 
suicide. In clinical settings, it is relatively straightforward to ask patients whether they have suicidal ideation, because this is one of the most important routine questions in mental health. ${ }^{6}$ Our previous study reported the prevalence of suicidal ideation $(30 \%)$ in a psychosomatic clinical setting. ${ }^{7}$ Naturally, attempted suicide is one of the strongest risk factors for death by suicide, ${ }^{8-11}$ and $60 \%$ of planned first attempts occur within the first year of ideation onset. ${ }^{9}$ Therefore, identifying those people with suicidal ideation would be logically the first step in reducing the incidence of suicide.

One problem is that, in screening process such as workplaces, it might be difficult to identify people with suicidal ideation because asking workers whether they had suicidal ideation could lead to conflicts in their relationships. In addition, people from Asian countries are sometimes reluctant to speak about their mental condition, including suicidal ideation, even to their physicians, because it is considered taboo. ${ }^{2}$ The Japanese in particular are less willing to express emotional distress or feelings such as suicidal ideation compared with Westerners. ${ }^{12} 13$ Therefore, we try to identify both suicidal ideation and the symptoms closely related to suicidal ideation, and their role in determining an individual's risk of suicide.

In Japan, more than 30000 people, 25/100 000 people, have committed suicide each year for the past 10 years, and $27 \%$ of them were working people. ${ }^{3}$ Thus, we thought that it was highly important to identify the prevalence of suicidal ideation and the symptoms that are closely related to suicidal ideation in the working population in Japan.

The aims of this study were twofold. First, we attempted to determine the prevalence of suicidal ideation in Japanese workers because of the lack of data at present on the prevalence of suicidal ideation in the working population. Second, we examined the symptoms of major depressive disorder (MDD) which could serve as predictors of suicidal ideation even in a nonclinical setting, hypothesising that some of these symptoms would be strongly related to suicidal ideation.

\section{METHODS}

\section{Participants and ethical considerations}

A cross-sectional design was used. Data were obtained from 1314 full-time office workers attending health examinations in an enterprise in Tokyo, Japan. Owing to missing health examination data, we excluded 48 participants and thus, we analysed the data from 1266.

Japanese workers $(96 \%$ of the total number of employees, 1100 men and 166 women aged 20-69 years). All participants provided written informed consent after receiving a complete explanation regarding the purpose, nature, and risks of the procedures used. This study was performed in accordance with the World Medical Association's Declaration of Helsinki, and its protocol was approved by the Ethics Committee of the
Teikyo University School of Medicine and the company's labour safety committee.

\section{Assessment of major depressive disorder including} suicidal ideation

To ascertain whether each participant met the Diagnostic Statistical Manual of Mental Disorders Fourth Edition, Text Revision (DSM-IV) ${ }^{14}$ criteria for MDD, clinical interviews for DSM-IV axis I disorders (Structured Clinical Interview for DSM-IV for axis I disorders: SCID-CV) were conducted by two study physicians specialising in both psychiatry and psychosomatic medicine, assisted by a researcher trained in psychology. Suicidal ideation was also evaluated during the interviews. To confirm the diagnosis of suicidal ideation, interviewees underwent additional independent interviews that specifically focused on suicidal ideation.

\section{Assessment of lifestyle factors}

A structured questionnaire administered by the physician was used to assess lifestyle factors, including smoking status, alcohol consumption and exercise. ${ }^{15}$ Based on their smoking history, the participants were classified into three categories: current smokers, past smokers and non-smokers (no history of smoking). Alcohol consumption was classified into three categories based on the weekly frequency of consumption: 67 times/week, 1-5 times/week and occasional drinking. Exercise was classified into two categories: regular or irregular. These questions were asked as additional questions during the DSM-IV interview.

\section{Sample size}

On the basis of our previous study, ${ }^{7}$ we expected to find a difference of $30 \%$ in the rate of each symptom between both groups with and without suicidal ideation. To achieve a power of 0.90 given a two-sided $\alpha$ of 0.05 , and a ratio of our exposed sample to our control sample of 1:25, we needed at least 32 participants in the group with suicidal ideation and 800 participants without suicidal ideation. Finally, through the actual data collection we collected 34 participants with suicidal ideation and 1232 participants without suicidal ideation.

\section{Statistical analysis}

After the characteristics of the study population were descriptively evaluated according to the presence of suicidal ideation, multiple logistic regression analyses were used to evaluate the association between suicidal ideation and each MDD symptom, by calculating crude and adjusted ORs. The ratios were adjusted for potential confounding factors to depression and suicide, such as sex, age and three lifestyle factors (smoking status, alcohol consumption and exercise). Finally, receiver operating curve analyses were performed to examine the predictive potentials of MDD symptoms for suicidal ideation. Statistical significance was inferred at $\mathrm{p}<0.05$ (two-tailed) for both the crude and adjusted ORs of the logistic 
analyses and the test equality of the area under the curve (AUC). All analyses were performed using STATA V.11.

\section{RESULTS}

A total of $34(2.7 \%, 95 \%$ CI (1.8 to 3.8$))$ and $70(5.5 \%$, $95 \%$ CI ( 4.3 to 6.8$)$ ) people were diagnosed with having suicidal ideation and MDD, respectively. Those with suicidal ideation were more likely to be aged between 40 and 49 years, drinkers and not exercising regularly (table 1). Only the age factor (ie, being in one's $40 \mathrm{~s}$ ) significantly differed between participants with and without suicidal ideation. Six of the eight symptoms of MDD (depressive mood, loss of interest, weight loss, psychomotor agitation, worthlessness and concentration loss) were related to suicidal ideation in both the crude and adjusted analyses (table 2).

Depressive mood had the strongest relationship to suicidal ideation (OR 11.2, 95\% CI (5.4 to 23.0)), followed by worthlessness (OR 8.5, 95\% CI (4.1 to 17.4)) and then concentration loss (OR 6.1, 95\% CI (4.1 to 17.4)).

Worthlessness had the highest AUC $(0.73,95 \%$ CI $(0.71$ to 0.76$))$ in predicting suicidal ideation, followed by concentration loss $(0.71,95 \%$ CI $(0.62$ to 0.79$))$ and then depressive $\operatorname{mood}(0.69,95 \%$ CI $(0.66$ to 0.71$))$.

\section{DISCUSSION}

Our study revealed that the prevalence of suicidal ideation was $2.7 \%$ in Japanese workers. In addition, we found that six symptoms of depression, particularly depressive mood, worthlessness and concentration loss, were related to suicidal ideation. These are potential predictors that can be used in identifying suicidal ideation among Japanese workers. As we expected, suicidal ideation was less prevalent in Japanese workers than in Western populations. ${ }^{16}$ We may have to consider that this low prevalence of suicidal ideation is not reflective of a low prevalence in the general population, because some Japanese people are reluctant to speak about their mental condition. While we will need to reconsider this prevalence rate in the near future, this rate is useful as one of the first evaluations of suicidal ideation among workers in Japan, which has one of the highest rates of completed suicide worldwide.

Although there is no way to predict who will eventually die by suicide, treating the clear warning signs for suicide can reduce patients' suffering. This study revealed a significantly positive association between some symptoms of depression and suicidal ideation. In countries like Japan, where people are reluctant to express their feelings, these symptoms might be useful in estimating an individual's risk of suicidal ideation and eventual suicide. ${ }^{416}$

Some of the above symptoms (depressive mood and worthlessness) are similar to symptoms found to be predictors of suicidal ideation in previous studies. ${ }^{16}{ }^{17}$ Because the findings of previous studies applied to clinical patients, our study generalised those symptoms as a predictor of suicidal ideation to the working population. Only concentration loss was a new predictor, which might be due to the characteristics of our study sample. For workers, concentration loss would be a particularly salient symptom due to its interruption of their daily work.

Our results also showed that while participants who were between 40 and 49 years old, drinkers and not

\begin{tabular}{|c|c|c|c|c|c|c|}
\hline \multirow[b]{2}{*}{ Variable } & \multicolumn{3}{|c|}{ Suicidal ideation + $(n=34)$} & \multicolumn{3}{|c|}{ Suicidal ideation $-(n=1232)$} \\
\hline & Per cent & $95 \% \mathrm{Cl}$ & Per cent & $95 \% \mathrm{Cl}$ & OR & $95 \% \mathrm{Cl}$ \\
\hline \multicolumn{7}{|l|}{ Sex } \\
\hline Male & 79.4 & (62.1 to 91.3 ) & 87.1 & (85.1 to 88.9 ) & 0.6 & (0.2 to 1.3$)$ \\
\hline Female & 21.6 & (8.7 to 37.9 ) & 12.9 & (11.1 to 14.9 ) & 1.0 & (1.0 to 1.0$)$ \\
\hline \multicolumn{7}{|l|}{ Age } \\
\hline $20-29$ & 14.7 & (2.6 to 26.8 ) & 9.3 & (7.6 to 10.9$)$ & 1.6 & (1.0 to 2.5$)$ \\
\hline 30-39 & 26.5 & (11.4 to 41.5 ) & 29.9 & (27.3 to 32.4 ) & 1.5 & (0.8 to 2.8$)$ \\
\hline $40-49$ & 47.1 & (30.0 to 64.1 ) & 29.9 & (27.3 to 32.4 ) & 4.2 & (1.4 to 12.5$)$ \\
\hline $50+$ & 11.7 & (0.4 to 23.2$)$ & 30.9 & (28.4 to 33.6 ) & 1.0 & (1.0 to 1.0$)$ \\
\hline \multicolumn{7}{|l|}{ Smoking status } \\
\hline Current & 38.2 & (21.6 to 54.8 ) & 36.4 & (33.6 to 39.1 ) & 1.0 & (0.7 to 1.5$)$ \\
\hline Past & 26.5 & (11.4 to 41.5 ) & 31.0 & (28.4 to 33.6 ) & 0.8 & (0.3 to 1.9$)$ \\
\hline Never & 35.3 & (19.0 to 51.6 ) & 32.6 & (30.0 to 35.3 ) & 1.0 & (1.0 to 1.0$)$ \\
\hline \multicolumn{7}{|l|}{ Alcohol consumption } \\
\hline Every day (6-7 times/week) & 29.4 & (13.9 to 45.0$)$ & 18.8 & (16.6 to 20.9 ) & 0.7 & (0.4 to 1.1$)$ \\
\hline Often (1-5 times/week) & 47.1 & (30.0 to 64.1 ) & 46.5 & (43.7 to 49.3 ) & 0.6 & (0.3 to 1.4$)$ \\
\hline No regular drinking & 23.5 & (9.0 to 38.0$)$ & 34.7 & (32.1 to 37.4 ) & 1.0 & (1.0 to 1.0$)$ \\
\hline \multicolumn{7}{|l|}{ Exercise } \\
\hline Irregular & 70.6 & (52.5 to 84.9$)$ & 62.1 & (59.3 to 64.8 ) & 1.5 & (0.7 to 3.1$)$ \\
\hline Regular & 29.4 & (15.1 to 47.5 ) & 37.9 & (35.2 to 40.7 ) & 1.0 & (1.0 to 1.0 ) \\
\hline
\end{tabular}




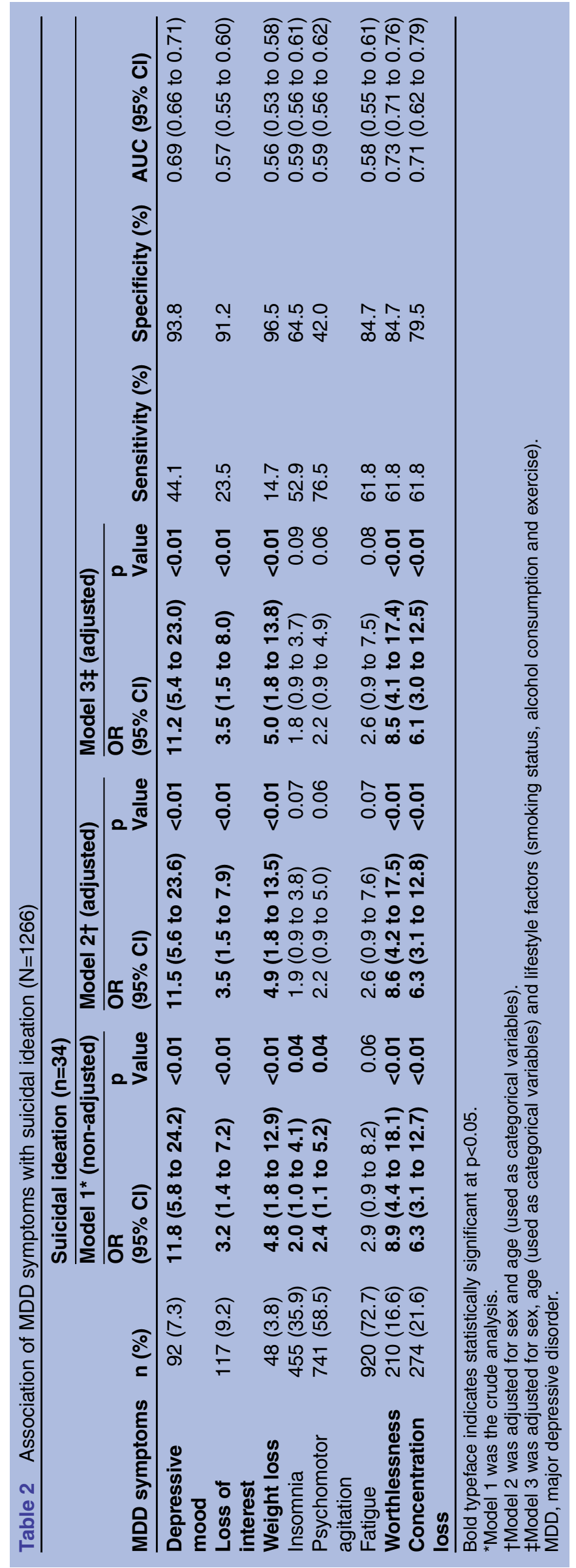

exercising regularly had a higher tendency for suicidal ideation, only being in one's 40 s was statistically significant in this study. This finding would be reasonable because the greatest increase in the number of deaths by suicide in the last decade in Japan occurred among 40-year-old to 49 -year-old workers. ${ }^{3}$ Although not statistically significant, heavy drinking has been shown to be associated with a higher risk of alcohol abuse and not exercising regularly is associated with a greater risk of poor mental health. ${ }^{1}$ We believe that completed suicides have a background of suicidal ideation. Thus, our recommended strategy to reduce suicide based on this research is to identify its potential risk by asking indirect questions related to suicidal ideation, including questions related to depressive mood, worthlessness and concentration loss, because these symptoms appear to be related to the presence of suicidal ideation, which in turn increases the likelihood of completed suicide.

Several limitations of this study warrant consideration. First, because of its cross-sectional nature, our results do not directly imply that symptoms of MDD and suicidal ideation are part of a clinical continuum, but rather that a simple statistical association exists after adjusting for important confounding factors. To confirm the results, a more robust study such as a prospective cohort study would be needed. Second, because of the small sample size, the results do not directly represent the general population of Japanese workers. The study is only applicable to a working population in an urban area in Japan. However, this is one of the first studies to report the prevalence of suicidal ideation in Japanese workers. Thus, this report could be of paramount importance in Japan, where many people are reluctant to discuss their mental condition.

Contributors TT and MN collected and analysed the data. TT was involved in writing the manuscript. MN reviewed and edited the manuscript and helped in its analysis. All authors contributed to the discussion.

Funding This research was supported in part by Grant-in-Aid for Scientific Research (2009-2012) (C) from the Ministry of Education, Science, Sports and Culture, Japan; and Research Grant (2011-2014) for Health and Welfare from Ministry of Health, Labour and Welfare, Japan (No. 24590761).

Competing interests None.

Provenance and peer review Not commissioned; externally peer reviewed.

Data sharing statement No additional data are available.

Open Access This is an Open Access article distributed in accordance with the Creative Commons Attribution Non Commercial (CC BY-NC 3.0) license, which permits others to distribute, remix, adapt, build upon this work noncommercially, and license their derivative works on different terms, provided the original work is properly cited and the use is non-commercial. See: http:// creativecommons.org/licenses/by-nc/3.0/

\section{REFERENCES}

1. World Health Organization. Suicide rates per 100000 by country, year and sex. 2011. http://www.who.int/mental_health/prevention/ suicide_rates/en/ (accessed 12 Jan 2013).

2. Nakao M, Takeuchi T. The suicide epidemic in Japan and strategies of depression screening for its prevention. Bull World Health Organ 2006;84:492-3.

3. National Police Agency. Japan's suicide statistics in 2010. http:// www.npa.go.jp/safetylife/seianki/H22jisatsunogaiyou.pdf (accessed 14 Jul 2013). 
4. Mcdowell AK, Lineberry TW, Bostwick JM. Practical suicide-risk management for the busy primary care physician. Mayo Clin Proc 2011;86:792-800.

5. Zhang Y, Law CK, Yip PS. Psychological factors associated with the incidence and persistence of suicidal ideation. J Affect Disord 2011;133:584-90.

6. American Psychiatric Association. Practice guidelines for the treatment of psychiatric disorders compendium 2004. Tokyo, Japan: Igaku Shoin, 2006:1-966.

7. Nakao M, Yamanaka G, Kuboki T. Suicidal ideation and somatic symptoms of patients with mind/body distress in a Japanese psychosomatic clinic. Suicide Life Threat Behav 2002; 32:80-90.

8. Takeuchi T, Nakao M, Yano E. Screening for major depression in the workplace: testing diagnostic accuracy of a two-item questionnaire used during mandatory testing. Prim Care Community Psychiatr 2006;11:13-19.

9. Kessler RC, Borgers G, Walters EE. Prevalence of and risk factors for lifetime suicide attempts in the National Comorbidity Survey. Arch Gen Psychiatry 1999;56:617-26.
10. Suominen $\mathrm{K}$, Isometsä $\mathrm{E}$, Suokas J, et al. Completed suicide after a suicide attempt: a 37-year follow-up study. Am J Psychiatry 2004;161:562-3.

11. ten Have M, De Graaf R, van Dorsselaer S, et al. Incidence and course of suicidal ideation and suicide attempts in the general population. Can J Psychiatry 2009;54:824-33.

12. Kleinman A. Neurasthenia and depression: a study of somatization and culture in China. Cult Med Psychiatry 1982;6:117-90.

13. Simon G, Von Korff M, Piccinelli M, et al. An international study of the relation between somatic symptoms and depression. $N$ Engl J Med 1999;341:1329-35.

14. American Psychiatric Association. DSM-IV-TR. Diagnostic and statistical manual of mental disorders. 4th edn. revised. Washington, DC: American Psychiatric Association, 2000.

15. Nakao M, Yano E. Prediction of major depression in Japanese adults: somatic manifestation of depression in annual health examinations. J Affect Disord 2006;90:29-35.

16. van Gastel A, Schotte C, Maes M. The prediction of suicidal intent in depressed patients. Acta Psychiatr Scand 1997;96:254-9.

17. Hawton K, van Heeringen K. Suicide. Lancet 2009;18:1372-81. 